\title{
Web Intelligent for Forecasting Exchange Rate Currency using Clever Extraction Agent Combine with Financial Data Mining
}

\author{
Khammapun Khantanapoka \\ Department of Multimedia Technology \\ Faculty of Science, Chandrakasem Rajabhat University, Bangkok, Thailand \\ doramon86@hotmail.com
}

Received: January 29, 2021. Revised: February 26, 2021. Accepted: March 15, 2021. Published: March 29, 2021.

\begin{abstract}
From the current economic climate results in fluctuations of currency exchange rates in all countries. Since the most countries use USD as the reference exchange rate. The exchange rate will change from day to day so variety of factors which affect the exchange rate forecasting in the exchange rates in advance are critical to evaluate for the impact of the economic system of each country. It is important for investment decisions, exports, and profitability in the money market. It was reported on website (www) in the daily exchange rate changes. We use clever search agent (CSA) gather information from financial website generate to financial data mining. Kohonen Neural Networks is the method to determine similarity of internet documents using pattern index of financial document. And Ontology Structure of Sentence is the method to determine keyword using pattern index of financial content. Both are important components of Financial Data Mining. It is analyzed for exchange rate forecasting about USD/ Pounds. Our experimental forecast exchange rates for currency's USD / Great Britain Pounds by compare three algorithms as fallows GA, Meiosis Genetic Algorithms (MGA). This research propose new algorithm is called Dash Predator Swarm Optimization (DP2SO) which are accurate in prediction than other methods in generation of Genetic algorithm (GA) 35.83-41.52\% which it depend on the accuracy of the information in each factor which are important finance dataset. It will present the future trends of exchange rate to the individual website.
\end{abstract}

Keywords- exchange rates; USD/Great Britain Pounds.

\section{INTRODUCTION}

Normally, the study of the financial market evolution and particularly exchange markets are considered as one of the most important research fields in international finance as long as exchange markets are considered as open systems which react on the base of collected information during the time periods. There are many factors to forecast exchange rates such as differentials in inflation, differentials in interest rates, current-account deficits, public debt, terms of trade, political stability and economic performance, monetary policy, political situation, balance of payment, market judgment. However, in the short-term will depend on six factors such as interest rate, trade flows, natural disasters, economic growth, link to commodity based currencies, conflict and short term inflation. The behavior modeling process of the exchange markets requires beforehand a deepen knowledge of the exchange system and factors which influence it. The major risk which an intervening party is dealing with is the non-linearity of exchange rate data sets. Thus, this study is articulated with the following problematic: the contribution of the application of artificial intelligence tools in the modeling of the agents' behavior intervening in a financial market while trying to improve their training processes for the prediction. It consists in applying a hybridization of two fundamental tools of artificial intelligence, the artificial neural networks (ANN) and the meiosis genetic algorithms (MGA).

\section{LITERATURE REVIEW}

Assessing the future changes in exchange rate is the main concern of policy makers and economist since it plays an important role in the economy [1]. However, the better understanding of the movements of exchange rate will provide the confidence for policy makers to keep inflation stable [2]. It is impotent factor of modern economic. Therefore application of nonlinear models becomes more important since nonlinear models can predict the time series variables with higher accuracy than linear models. Artificial intelligence (AI) is widely accepted as a nonlinear models for time series forecasting as they can learn the past behavior of variables and recognize the complexity and nonlinearity in the pattern of data set. AIs can be applicable in robotics, medicine, pattern recognition, forecasting, manufacturing, power systems, optimization, etc [3].

\section{A. Financial Data Mining : Intelligent Search Agent for Internet Document}

We find impotent information from financial website. We building pattern index of financial document from keyword and use search internet document from website of banking and financial resources. We use Kohonen Neural Networks is the method to determine similarity of internet documents on internet network. Kohonen Neural 
Networks decrease operation for convert to quantitative data while protect context of the original data.

Kohonen Neural Networks developed for learn to classify data without supervision. With this approach an input vector is presented to the network and the output is compared with the target vector. If they differ, the weights of the network are altered slightly to reduce the error in the output. This is repeated many times and with many sets of vector pairs until the network gives the desired output. Kohonen Neural Networks Text search consists of input unit and output unit $\mathrm{X}=\left(x_{1}, x_{2}, \ldots, x_{\mathrm{d}}\right)^{\mathrm{T}}$

while $\mathrm{W}_{\mathrm{i}}=\left\{w_{i 1}, w_{i 2}, \ldots, w_{i k}\right\}$. We apply the concept of conscience algorithm combine with search difference of whole documents. We use operation search for wining neural of competitive learning. We can find difference value of feature $x_{k}$ and weight vector $w_{i k}$ from equation:

$$
D\left(x_{k}, w_{i k}\right)=D_{s}\left(x_{i}, w_{i k}\right)+D_{T}\left(x_{i}, w_{i k}\right)
$$

by difference value for span

$$
D_{s}\left(x_{i}, w_{i k}\right)=\frac{\left|L\left(x_{k}\right)-L\left(w_{i k}\right)\right|}{\operatorname{Span}\left(L\left(x_{k}\right) \operatorname{and}\left(w_{i k}\right)\right)}
$$

by difference value for content

$$
D_{T}\left(x_{i}, w_{i k}\right)=\frac{\mid L\left(x_{k}\right)+L\left(w_{i k}\right)-2 * L\left(\text { intersection }\left(x_{k}, W_{i k}\right)\right) \mid}{\operatorname{Span}\left(L\left(x_{k}\right) \operatorname{and}\left(w_{i k}\right)\right)}
$$

where $\mathrm{L}$ as Length

We choose node which neural output $w_{i}$ which has The difference is minimal.

First step, initialize weight $w_{i k}$ in each neural output $\mathrm{w}_{\mathrm{i}}$. Each weight can be initializing from the training data arbitrarily by $\mathrm{W}_{\mathrm{i}}=\left\{w_{i 1}, w_{i 2}, \ldots, w_{i k}\right\}$

$$
w_{i k}\left\{\left(A_{i k 1}, e_{i k 1}\right),\left(A_{i k 2}, e_{i k 2}\right), \ldots,\left(A_{i k p}, e_{i k p}\right)\right\}
$$

where $\mathrm{w}_{\mathrm{ik}}$ as Weight of neural $\mathrm{w}_{\mathrm{i}}$ for feature $k$

$$
\begin{aligned}
& A_{i k p} \text { as Member of } \mathrm{w}_{\mathrm{ik}} \\
& e_{i k p} \text { as Degree of membership of } A_{i k p}
\end{aligned}
$$

Second step, while stopping condition is false then operation step 2-6

Third Step, draw a sample $X$ from the input distribution with a certain probability. Each input vector of Number of features $\mathrm{X}=\left(x_{1}, x_{2}, \ldots, x_{\mathrm{d}}\right)^{\mathrm{T}}$, then operation step 4-6

Forth step 4, each output unit $i$ to compute

$$
\left\|X-W_{i}\right\|=\sum_{k=1}^{d} D\left(x_{k}, w_{i k}\right) * E \text { which } E=1-\frac{\sum e_{i k p}}{D}
$$

where $d$ as number of feature and $\mathrm{E}$ is defined by

$$
A_{i k p} \in W_{i k} \cap x_{k}, e_{i k p} \text { is degree of membership of }
$$

$A_{i k p}$ and $\mathrm{D}=\sum e_{i k}$ by look for index $i$ such that $\left\|X-W_{i}\right\|$ is minimum.

Fifth step, keep a running total of the fraction of time $P_{i}$ which neural $W_{i}$ win the competition. We find total of the fraction of time with this equation.

$$
P_{i}^{\text {new }}=P_{i}^{\text {old }}+B\left(y_{i}-P_{i}^{\text {old }}\right)
$$

where $0<\mathrm{B}<1$ and if neuron $i$ is wining neuron $y_{i}=1$

but otherwise $y_{i}=0$

Sixth step, search the wining neuron using conscience mechanism

$$
\left\|X-W_{i}\right\|=\min \left(\sum_{k=1}^{d} D\left(x_{k}, w_{i k}\right) * E-b_{i}\right)
$$

where $b_{i}$ is defined by $b_{i}=C\left(\frac{1}{N}-P_{i}\right)$

where $C$ is bias factor and $\mathrm{N}$ is total number of neurons in the network. We want bias term.

Seventh step, for all weight that connect to the winning node $i$ and neighborhood $\left(\wedge_{i}\right)$ It will adjust weight vector to most nearby input $X$, and update $A_{i k p}$ of $w_{i k}$ for new member.

$$
W_{i k}^{\text {new }}=\left\{\begin{array}{c}
W_{i k}^{\text {old }} \cup x_{k} \quad \text { if } \quad i \in \wedge_{i} \\
W_{i k}^{\text {old }} \text { otherwise }
\end{array}\right.
$$

We improve $e_{i k p}$ of $w_{i k}$ three cases such as increase, lower and appropriate.

$$
e_{i k p}^{\text {new }}=\left\{\begin{array}{ccc}
f\left(e_{i k p}^{\text {old }}+\eta\right) & \text { if } & A_{i p k} \in w_{i k} \cap x_{k} \\
f\left(e_{i k p}^{o l d}-\eta\right) & \text { if } & A_{i p k} \notin w_{i k} \cap x_{k} \\
2 * \eta & \text { if } & A_{i p k} \in x_{i}-\left(w_{i k} \cap x_{k}\right)
\end{array}\right.
$$

where $f$ is defined as below

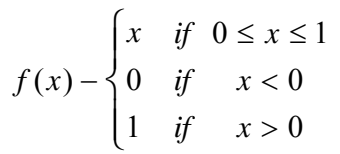

Eighth step, backward step 2-7 unit the stopping condition is true.

\section{B. Financial Data Mining : Ontology Structure of Sentence}

The word ontology has been given many definitions both philosophy and information technology. The meaning of ontology of information technology means the explanation of the concept according to interested scope or the specification of conceptualization. The ontology is the creation of knowledge structure in any aspect or any domain which has the same concept and understanding. Ontology is used in explaining the meaning of various things and can categorize the internet documents of information in one interested scope. We determine word 
using pattern index of financial content. We use two level as follow Financial keyword and and Command Tag.

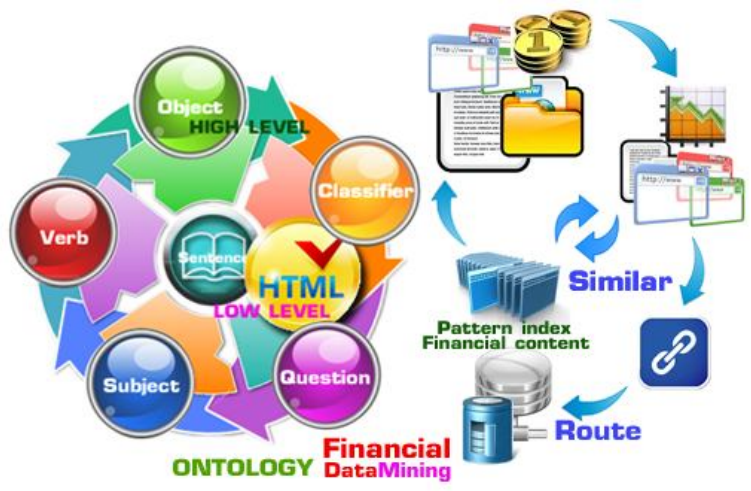

Figure 1. show pattern index of financial content

Lower Level Catalog(LLC) of content such as $<\mathrm{a}>$ $<$ abbr $><$ address $><$ area $><$ article $><$ aside $><$ audio $><$ b $><$ ba se $><$ bb $><$ bdi $><$ bdo $><$ blockquote $><$ body $><$ br $><$ button $>$ $<$ select $><$ small $><$ source $><$ span $><$ strong $><$ style $><$ sub $><$ summary $><$ sup $><$ table $><$ tbody $><$ td $><$ textarea $><$ tfoot $>$ $<$ th $><$ thead $><$ time $><$ title $><$ tr $><$ track $><$ u $><$ ul $><$ var $>$,etc. High Level Catalog (HLC) of content such as exchange rate, interest rate, balance sheet, bank loans, Bonds lend, governments money, different lengths of time, maturity date, bondholders, coupon of a bond, inversely related, Market interest rates, current price ,bond pays, yield curve, borrowing changes, different maturity, investors expect, interest exchange rate of owner's equity, economic indicator flexible of demand, MXN USD GBP EUR JPY MYR SGD BND PHP IDR INR CHF AUD NZD PKR, etc. It returns token link to server that we generate to financial data mining. We define Pattern component as following : $\mathrm{S}$ as subject $\mathrm{V}$ as verb, $\mathrm{vp}$ as verb phrase, $\mathrm{Cl}$ vp as adverb used to exemplify verb phrase, $\mathrm{O}$ as object, $\mathrm{Cl}$ as Adverb phrase that exemplifies noun which includes the adverb telling the amount and noun, $\mathrm{Cv}$ as Adverb phrase that exemplifies adverb phrase. Q as Question, Classifier as word for connecting between sentences (FANBOY).We separate three mode as follow : mode 0 , it can be anything that is not repeated. mode 1 , it must be the same type for the entire sentence. mode 2, it must be in the same category.

Example, Checking a sentence of addition Pattern Mode
$\mathrm{S}+\mathrm{V}+\mathrm{O}+\mathrm{Cl}+\mathrm{V}+\mathrm{O}+\mathrm{Cl}+\mathrm{Num}$
Code pattern is $2: 1: 2: 1: 0$
$\mathrm{S}+\mathrm{V}+\mathrm{O}+\mathrm{Cl}+\mathrm{Num}+\mathrm{O}$
$\mathrm{S}+\mathrm{Cl}+\mathrm{V}+\mathrm{Cl}+\mathrm{O}$
Code pattern is $1: 1: 2: 2: 0$
$\mathrm{S}+\mathrm{V}+\mathrm{O}+\mathrm{Cl}+\mathrm{vp}+\mathrm{O}$
$\mathrm{S}+\mathrm{V}+\mathrm{O}+\mathrm{Cl}+\mathrm{vp}+\mathrm{Cl}+\mathrm{Q}$
Code pattern is $1: 0: 1: 1: 0$
Code pattern is $1: 1: 1: 0: 0$
Code pattern is $1: 1: 1: 0: 0$

\section{Artificial Neural Network (ANN)}

An Artificial Neural Network (ANN) or also knows as Neural Network (NN) is a mathematical model which is processing information with computing by connectionist to simulate the operation of neural networks in the human brain. With the objective of creating tools that are able to learn pattern recognition and knowledge deduction as well as the ability of the human brain. The concept was beginning of this technique is derived from the study of bioelectric network in the brain, which contains nerve cells, or neurons and the coordination of nerves (Synapses),

Normally, the neural networks have 4 elements which are; 1) Processing Unit; This unit can be divided into 3 parts, which are Input unit: to receive data from external, Hidden unit: to convert Internal data and output units, Output unit: to decision or control signals. 2) Connections, the processing units occurred in neural networks, which will be organized into various structures via connection.

3) Computing Procedure is a standard process which is based on every neural network. 4) Training Procedure, is a process which modify the connection weight, or in case of fine structure in neural networks in order to add or delete the connection node. Weight change the connection looks more general restructuring. Because of the connection weight values equals to zero, it represents a connection which is removed from neural networks. However, the changing structure of the neural network will increase the speed of learning and increase the ability to recognize common patterns. However, factor of exchange rate determinants has sixteen variables both the economic and financial parameter in ANN model as (1) sign of exchange rate return of the previous period (2) consumer price level (3) inflation rate (4) short-term interest rate (5) change in balance of payment. We use partial least squares regression for initial value in start epoch of neural network.(ANN) Partial least squares is one solution for optimum problems. This method manages principle factor extraction.

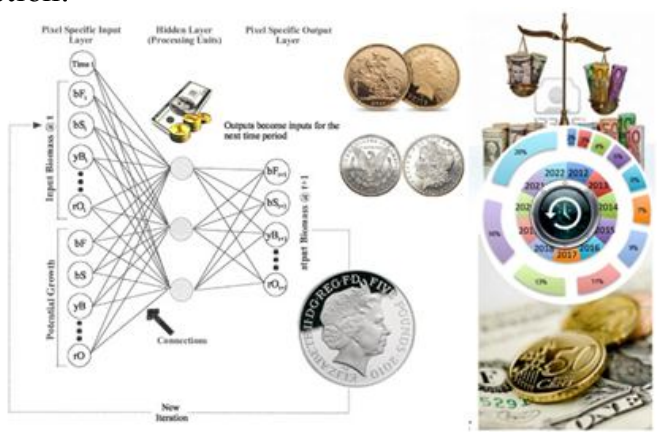

Figure 2. Artificial Neural Network Framework

\section{Meiosis Genetic Algorithm}

Genetic algorithm [1] is a technique which is used to find out the principles of evolutionary theory [2], instead of answers in chromosome. This algorithm have a fitness function of chromosomes with problems in finding the right answer of the algorithm is based adaptation of the chromosome to the specific problems through the process four process, i.e. selection, crossover, mutation and its progeny to their parents instead of models. Which the main problem of this algorithm is a problem in the convergence speed to answer and problems of convergence to an incorrect answer or hill climbing 
problem. To resolve the speed of convergence to answer, Maeda [3] brought the theory of inequality each of evolution [4] to refine genetic algorithms to produce chromosome progeny that are similar as parent models. For solving the problem of convergence to an incorrect answer, Ronald [5] has improved the genetic algorithms by removal chromosome that looks unique from the system, to produce chromosome diversity which increases the chance to enter the correct answer.

When we are considering the Meiosis cell division, it was found that chromosome which contains both of products chromosome inherited property from their parents' generation and different chromosome from their parents. The new product range with two chromosomes make genetic algorithms that mimic cell division, this is convergence to the correct answer quickly. The Meiosis cell division [6] is divided into reproductive cells for using in the breeding of organisms. During Meiosis 1, the number of cells will increasing which key processes of Meiosis cell division consist of a replication of the chromosome itself. The occurrence of mutations and the crossover are shown by the following details. Simulation of the chromosome itself is divided into two types which are the simulation itself as an equal and as an unequal [4]. Because the functional group of base on chromosomes in the lagging cannot simulation itself continuous therefore it's caused the connection between the functional group of base, called "point of joining" where the errors in reading data from the original chromosome has more than $20-30 \%$ so these lines are different from the original chromosome. The mutations are occurred by a wrong reading of the original chromosome in the process of simulation itself. The production of chromosomal changes from the original. Crossover is occurred by the interlacing of chromosome.

\section{EXPERIMENTIAL OF PREDECTION}

\section{E.Classification of factor with Artificial Neural Network}

The ANN contains three layers which are an input layer, an output layer and one or more intermediate layers called hidden layers. Each layer consists of a number of units called neurons which are provided with activation functions (transfer functions). These will vary from one network to another which depends on the number of units in each layer, the number of weights, the layers arrangement and the activation function which are assigned to each layer. A neural network structure is defined by the various previous works. The typical activation functions include the sigmoid function, the tangent function, the hyperbolic function and the sinus and cosines functions. We note that there are no rules to select the activation function type, the choice is made arbitrarily. The sigmoid function is generally the most adaptation knowledge which is using different types of activation function has no major effect on network performance. The ANN functioning is similar to the human brain, because it captures the relationship between the variables after a representative set of data or examples while is considering the principle of induction, which is called learning by experience. The ANN content generally gives a simple no description of the problem. Hence, this method provides an alternative conventional analytical technique which is often limited by strict assumptions of normality, linearity and independence of variables. However, the ANN does not always give us a usable rule by a human. This system often remains a black box which provides a response for a given presented data, but it doesn't offer an easily interpretable justification. The first step of learning procedure is calculating the optimal weights of the various synaptic connections using a sample data. We describe the most commonly learning technique which is used is the back propagation. Before learning phase of neural system has been started, it should first proceed by normalization of the raw data which is considered as the most important pretreatment when using ANN. The most common method is expressed by the following equation:

$$
\mathrm{X}_{n}=\frac{\mathrm{x}_{i}-\mathrm{x}_{i, \min }}{\mathrm{x}_{i, \text { max }}-\mathrm{x}_{i, \text { min }}}\left(\mathrm{h}_{t}-\mathrm{l}_{t}\right)+\mathrm{l}_{t}
$$

Where $x_{n} \quad$ is the given normalized value,

$\mathrm{X}_{\mathrm{i}}$ is the original data value,

$\mathrm{X}_{i, \text { min }}$ is the minimum value in the dataset.

$\mathrm{X}_{i, \text { max }}$ is the maximum value in the dataset.

$\mathrm{h}_{\mathrm{t}} \quad$ is the upper bound of standardized data

$1_{t}$ is the lower bound of standardized data

By applying this transformation, we can conduct all the variables in order to have the same orders of magnitudes, which facilitate the determination of standard values for the algorithm parameters. This allows to obtain satisfactory results without adjusting them for each new problem and also to compare the distributions of variables. The general principle of the feed-forward algorithm is to give the network a large number of examples for the input and output associated which are known and weights are modified to correct the error committed by the network (i.e. the difference between the desired and the obtained responses). Thus learning is seen as an optimization problem of finding the network coefficients for minimizing a cost function. The most used cost function is the squared function on the base of learning; it is to minimize the sum of squared errors between the network output and the real value of the output. The cost function at the iteration defined on the entire training set is as follows:

$$
\mathrm{E}_{\mathrm{t}}=\frac{1}{\mathrm{~N}} \sum_{\mathrm{t}=1}^{\mathrm{n}}\left(\mathrm{y}_{\mathrm{t}}-\mathrm{t}_{\mathrm{t}}\right)^{2}
$$

where $E_{t}$ is the mean square error

$\mathrm{N}$ is the sample size

$y_{t}$ is the vector of desired outputs

$t_{t}$ is the vector of network outputs for iteration ( $t$ )

One of the main ANN advantages is that their learning 
algorithms are applicable to all networks types. We have all the freedom as regards the choice of the best adapted architecture to the problem, and whatever the network structure, we can always use the same set of learning algorithms. This flexibility allows implementation including networks whose architecture depends strongly on the structure of the problem to model expressed by equations. The problem that appears most often during learning is the over-fitting. If the ANN learns insufficiently or non-appropriately, it will give incorrect results when it receives some data slightly different. To avoid over-fitting, the performance of the trained network should then be compared on another set called validation set. This strategy should provide a better generalization of the model. This consists to monitoring the cost function evolution on a validation database and to stop the iterations when the calculated cost on this database begin to grow. Once the network is executed, we should always apply the tests to verify if it responds correctly. In the test phase, a sample part is simply removed from the training sample and conserved for the out-off-sample tests. For example, we can use $60 \%$ of the sample for learning, $20 \%$ for validation and $20 \%$ for testing.

\section{F. Create diversity of data with Meiosis Genetic Algorithms (MGA)}

Meiosis Genetic Algorithms are based on cell division, meiosis which was designed so that chromosomes are inherited property from their parents' generation. Both Chromosome and production are cover the scope of the problem. By adding a process model of an unequal chromosome and determined the mutations before the crossover and that the overalls process of Meiosis Genetic Algorithms based on meiosis cell division also include selected strain, chromosome simulation, mutations, crossover and selection of progeny to parents instead of models which each step is following work. The MGA has been designed so that chromosome progeny can inherit property from their parents' generation, by selection strains which are used to select and delete them. When the model parent chromosome are selected to produce progeny. After that they will remove the chromosomes which are selected by the system. Simulation of chromosome is using models for simulation by unequal chromosome because the chromosomes can produce a second chromosome where the first has ability to inherit property from their parents' generation which is called the Leading and the chromosome II. Capable of rapid evolution is called the lagging, It increase opportunities to find the best answer even more. Mutations occur only in the leading. Crossover has 2 point to increase the diversity of chromosome. The progeny instead model their parents because the MGA will produce progeny double model parents can be selected progeny of the replacement model parents of much of the generation parents selected. Chromosome specific child appropriate than previous time.

\section{G. Dash Predator Swarm Optimization $\left(D P^{2} S O\right)$}

We design and develop Predator Swarm Optimization Algorithm base on genetic algorithm for analysis search for optimal path base on several condition of animal which is predator. It consider from direction of each victim which is in front side. The victim tries avoiding congested pathway which cause to slowly movement. It catch slowly victim and follow excellent victim. It adjusts oneself to small size and extreme speedily. Seven Operation of Gene in Dash Predator Swarm Optimization as following ; 1) Simple Cross - Simple cross is a simple one point crossover between two of arrays of angles representing each path. 2) Subtree Cross - Subtree cross is also a one point crossover. A node is chosen in each binary tree and the nodes as well as their children are swapped between the two trees. Another variant of this operator swaps the two subtrees in the same location in both trees. This crossover is better than the simple cross. Each subtree, not consecutive values in the array holding the tree, represents a segment of the path. Using the subtree cross with the same cross site in both trees is equivalent to exchanging a portion of the two paths. 3)Random Mutation -This mutation randomly selects one node in the binary tree and replaces its value with a random angle.4) Small Mutation-This mutation randomly selects one node and perturbs the angle contained. This operator is performed more and more frequently as the number of generations increase and serves to fine tune an otherwise good path. 5) Flip Mutation -This mutation randomly selects one node and makes a convex segment of the path concave or vice versa. This mutation can be used to target path segments that intersect with obstacles and perturb them into free space. 6) Proportionate Selection - This operator propagates paths from one generation to the next giving each path a number of copies proportionate to its fitness compared to other paths and 7) Tournament Selection - This operator propagates paths from one generation to the next by selecting the best path of from a number of randomly paths.

\section{H. Fitness Function of Predator Swarm Optimization}

A good chromosome; when considering two chromosomes the better one is defined as the choices where less distance on requirement value to the goal. Keeping these two rules in mind, a suitable fitness function can be expressed as equation :

$$
f(\mathrm{p})=\mathrm{a}_{1} ? \delta(\mathrm{p})+\mathrm{a}_{2} \sum_{\mathrm{O}_{\mathrm{i}} \notin \mathrm{O}} \mathrm{C}\left(\mathrm{p}, \mathrm{o}_{\mathrm{i}}, \Delta\right)
$$

The first term is simply a scaled version of requirement value of the entire chromosome. In two dimensions, this can be calculated by summing the Euclidean norms of all the path segments comprising a chromosome as shown in below equation: 


$$
\delta(\mathrm{p})=\sum_{\mathrm{p} \notin \mathrm{P}} \sqrt{(\Delta \mathrm{y})^{2}+(\Delta \mathrm{x})^{2}}
$$

The second term of (4) is a penalty function to assess against value intersecting chromosome. It is computed by counting the number of collisions between the choice and all chromosomes. This fitness can easily be extended to penalize choice for chromosome or modified to appropriate choice. We determine base on fitness function in evolution of GA.

First step is generating chromosome process. We generate vector of probability value (p) which initial of each position as 0.5 . We use simulation gene inside chromosome with vector pattern of probability. It is cause of use memory less than genetic algorithm which can generate to circuit of hardware.

Second step is selecting chromosome. It is selecting chromosome of parents (father and mother generation) for generate chromosome in next generation base on principle of selection and delete.

Third step is defining parameter of $\mathrm{P}^{2} \mathrm{SOA}$

Fourth step is simulating chromosome. The chromosome will copy oneself to two chromosomes. A chromosome will controlled for non-mutation or mutate at least. It called leading. A chromosome mutate free from original chromosome. We call lagging. This method is effect to quickly search for optimum answer.

Fifth step is mutation in local search process. It is genetic variation effect to particularities of chromosome improved. We define mutate rate is stable and happen before the crossover process.

Sixth step is crossover in local search process. It is alternate gene by crossover for leading. It returns several chromosomes and cover every answer. While crossover for lagging which inherit hereditary characteristic from parent generation. The child chromosome has amount of chromosome more than double of parent. We must control number of chromosome which expands bit rapidly. We select child chromosome only high suitability for replace parent chromosome for next generation until altogether every chromosome.

Seventh step is compressed and compact chromosome and decompress. It help decrease rate of send data for calculation and increase speed in processing. We compress whole chromosome before consider in optimal of fitness function. It helps decrease size of data in temporary storage which has effect to decrease time in send data system than cGA. We use compress data with repeat data detection inside a part of chromosome. Example of compress chromosome, the pattern of repeat data composes three sections.

\begin{tabular}{|c|c|c|c|}
\hline $\begin{array}{c}\text { Compressed data } \\
\text { block 1 }\end{array}$ & $\begin{array}{c}\text { Compressed data } \\
\text { block 2 }\end{array}$ & $\cdots$ & $\begin{array}{c}\text { Compressed data } \\
\text { block n }\end{array}$ \\
\hline
\end{tabular}

Figure 3. show chromosome which compressed

\begin{tabular}{l|l|l}
\hline Length of repeating value & Repeat data count & Repeating value
\end{tabular} Figure 4. Show Set of repeat data $n$
They use four bits for Length of repeating value and four bits for Repeat data count. Bit of Repeating value depends to length of repeat data. Example by 10000001000000 1000000 use twenty one bits which generate from 1000000 repeat three times. It uses storage only fifteen bits which is enough for accuracy and robust information.

\begin{tabular}{|c|c|c|}
\hline 0111 & 0011 & 1000000 \\
\hline (a) & (b) & (c)
\end{tabular}

Figure 5. Show (a) Length of repeating value has7 bits, (b) Repeat data count data has 3 area, (c) Bit Repeating value is 1000000 by when decompress chromosome will return original data 100000010000001000000 .

As shown in Figure 6. the compressed chromosome consists of compressed data blocks. Each block has 3 fields: length of repeating value, repeat count and repeating value. The chromosome needs to be decompressed before the fitness evaluation starts. The length of the decompressed chromosome is varied. If the length of decompressed chromosome is longer than the size of the solution encoding, the excess string is discarded. If the length is less than the size of the solution encoding, the zero bits are added. The decompressed processing will decode one set of bit repeating value per times. Example, chromosome01000010100000110111100 which compose repeat data two sets $(0100000101000$ and 00110111100).

\begin{tabular}{|c|c|c|c|c|c|}
\hline (a) & 010000101000 & Repeat analysis to $>$ & 0100 & 0010 & 1000 \\
\hline (b) & 00110111100 & Repeat analysis to $>$ & 0011 & 0111 & 100 \\
\hline
\end{tabular}

Figure 6. Show two sets of repeat data (a) show frist set that bit repeating value is 1000 , Length of repeating value has 4 bits or $0100_{2}$ bits, Repeat data 2 times or $0010_{2}$ times. (b) show second set that bit repeating value is 100 , Length of repeating value has 3 bits or $0011_{2}$ bits, Repeat data 7 times or $0111_{2}$ times.

When dataset of (a) and (b) decompressed become to a original manuscript chromosome. Next step, we measure appropriate value of chromosome which decompressed. We check for each bit of chromosome by compare appropriate value and search for chromosome which has appropriate value more than on the other hand. We called chromosome of victor (winner). The chromosome which has appropriate value less than on the other hand, we called chromosome of loser.

If victor chromosome difference from loser chromosome then adjust probability value as follows;

Case One : if order bit $\mathrm{i}$ of victor is 0 use equation as $P[i]=p[i]+1 / n$

Case Two : if order bit $\mathrm{i}$ of victor is 1 use equation as $\mathrm{P}[\mathrm{i}]=\mathrm{p}[\mathrm{i}]-1 / \mathrm{n}$

where $\mathrm{n}$ is size of population

We will use compare fitness function for optimal answer.

Eighth step is calculation for fitness function. The properties of chromosome measured adapting to answer

Ninth step is comparison for fitness function in each round. We compare each fitness function in the same round. Next, we keep best chromosome which has distance between fitness function and answer at least in 
this round. It used compare with best chromosome in next round until found best parameter of answer.

Tenth step is turn back to third step until every parameter. Finally, we compare between best parameter of every parameter and choose best chromosome become to answer of problem. We will check status for found appropriate value of answer or not. Because, the condition in vector of probability will tendency 0.0 or 1.0 which can not guarantee found answer. Especially, In case of size of population $\mathrm{n}$ is a little value. It is effect to adjust a great number of values in each round rapidly. It is important consider effect to not found answer.

Case No ; the repeat operation in second step until found appropriate value of answer. Case Yes ; stop operation.

This model decides high accurately with local Search which improves from Particle Swarm Optimization(PSO). It is rapidly with several genes as double from gene object which Improved together with compress set of repeating genes which increase speed in calculation process.

\section{RESULT OF EXPERIMENTAL}

In this section, we will describe our experimental results of the Artificial Neural Networks integrate with Genetic Algorithms (ANN \& GA), Artificial Neural Networks integrate with Meiosis Genetic Algorithms (ANN \& MGA) and Artificial Neural Network integrate with Dash Predator Swarm Optimization (ANN \& $\mathrm{DP}^{2} \mathrm{SO}$ ) The results of predicting USD/GBP currency exchange rate using ANN \& $\mathrm{DP}^{2} \mathrm{SO}$ model are given in tables I and table II. From them we can see that using our ANN \& $\mathrm{DP}^{2} \mathrm{SO}$ to forecasting the USD/GBP exchange rate is better than all the models based on the criteria of RMSE and MAPE. When using the ANN \& $\mathrm{DP}^{2} \mathrm{SO}$ model for forecasting, we found that it is working high accurately while rapidly processes follow conceptual as small, rapidly and high accurately.

TABLE I. RESULT OF CLASTERING FINANCEIAL INFORMATION WEBSITE : REUTER $1 \& 2$

\begin{tabular}{|c|c|c|}
\hline $\begin{array}{l}\text { Internet document } \\
\text { Content (Reuter\#1) }\end{array}$ & $\begin{array}{l}\text { Website } \\
\text { (Reuter\#1) }\end{array}$ & $\begin{array}{c}\text { Precision Accuracy } \\
\text { (Reuter\#1) }\end{array}$ \\
\hline Exchange Rate & 121 & 96 \\
\hline Currency Climate & 257 & 224 \\
\hline Report Exchange & 59 & 53 \\
\hline $\begin{array}{l}\text { Internet document } \\
\text { Content (Reuter\#2) }\end{array}$ & $\begin{array}{l}\text { Website } \\
\text { (Reuter\#2) }\end{array}$ & $\begin{array}{c}\text { Precision Accuracy } \\
\text { (Reuter\#2) }\end{array}$ \\
\hline Exchange Rate & 121 & 84 \\
\hline Currency Climate & 257 & 241 \\
\hline Report Exchange & 59 & 47 \\
\hline
\end{tabular}

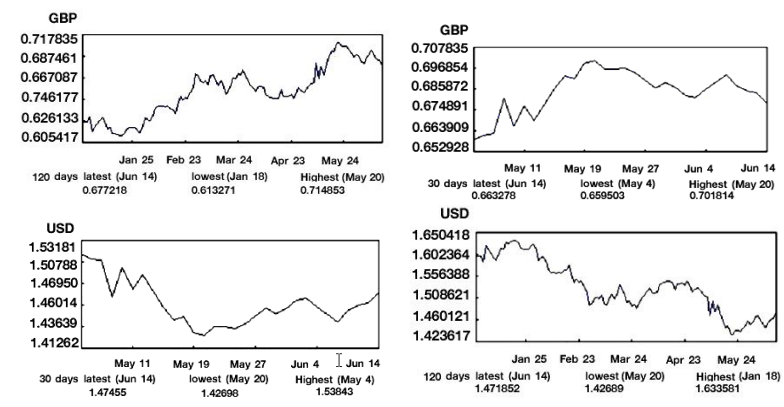

Figure 7. The average exchange rate of USD per 1 GBP in 30 days and 120 days ago.

TABLE II. DAILY USD/GBP CURRENCY RATE

\begin{tabular}{|c|c|c|}
\hline Model /Algorithm & RMSE $(* 100)$ & MAPE \\
\hline $\begin{array}{c}\text { ANN } \\
\text { Genetic Algorithms }\end{array}$ & 1.1437 & 0.8311 \\
\hline $\begin{array}{c}\text { ANN \& Meiosis } \\
\text { Genetic Algorithms }\end{array}$ & 0.9093 & 0.6173 \\
\hline ANN \& DP'SO & 0.7185 & 0.4336 \\
\hline
\end{tabular}

TABLE III. WEEKLY USD/GBP CURRENCY RATE

\begin{tabular}{|c|c|c|}
\hline Model /Algorithm & RMSE $(* 100)$ & MAPE \\
\hline $\begin{array}{c}\text { ANN } \\
\text { Genetic Algorithms }\end{array}$ & 1.8343 & 0.9899 \\
\hline $\begin{array}{c}\text { ANN \& Meiosis } \\
\text { Genetic Algorithms }\end{array}$ & 1.7077 & 0.9752 \\
\hline ANN \& DP'SO & 1.4486 & 0.6494 \\
\hline
\end{tabular}

V. CONCLUSION

According to the table, it shows compare three model that The ANN integrate with Genetic Algorithms, ANN integrate with Meiosis Genetic Algorithms and ANN integrate with Dash Predator Swarm Optimization $\left(\mathrm{DP}^{2} \mathrm{SO}\right)$. The result is found Dash Predator Swarm Optimization model provides high better performance. We use $\mathrm{DP}^{2} \mathrm{SO}$ forecast other currency exchange rate. However, we use these methods present investment on a personal website.

\section{REFERENCE}

[1] J. Groen, " Exchange rate predictability and monetary fundamentals in a small multi-country panel," Journal of Money Credit Bank, vol. 37, pp. 495-516, 2005.

[2] C. Pandaa and V. Narasimhanb, "Forecasting exchange rate better with artificial neural network," Journal of Policy Modeling, vol. 29, pp 227-236, 2007

[3] Z. Haoffi, X. Guoping, Y. Fagting, Y. Han, "A Neural Network Model Based on the Multi-Stage Optimization Approach for ShortTerm Food Price Forecasting in China," Expert Systems with Applications, Vol. 33, pp. 347-356, 2007.

[4] A. H. Shaari, B. gharleghi, K. Omar, T. Sarmidi, "Comparison of linear and nonlinear models for exchange rate forecasting," in Proc. 2 International Conference on Organizational Innovation, Malaysia, 2011, pp. 1792-1800.

[5] G. M. Mendez, "Orthogonal-back propagation hybrid learning algorithm for type-2 fuzzy logic systems," IEEE Proceedings of the NAFIPS 04 International Conference on Fuzzy Sets, June 2004, vol. 2, pp. 899-902.

[6] H. A. Hagras, "A hierarchical type-2 fuzzy logic control architecture for autonomous mobile robots," IEEE Transactions on Fuzzy Systems, vol. 12, No 4, 2004, pp 524-539.

Creative Commons Attribution License 4.0 (Attribution 4.0 International, CC BY 4.0)

This article is published under the terms of the Creative Commons Attribution License 4.0

https://creativecommons.org/licenses/by/4.0/deed.en_US 